\title{
Article
}

\section{Laserlight visual cueing device for freezing of gait in Parkinson's disease: a case study of the biomechanics involved}

Egerton, C. J., McCandless, P., Evans, B., Janssen, J. and Richards, Jim

Available at http://clok.uclan.ac.uk/12595/

Egerton, C. J., McCandless, P., Evans, B., Janssen, J. and Richards, Jim ORCID: 0000-0002-4004-3115 (2015) Laserlight visual cueing device for freezing of gait in Parkinson's disease: a case study of the biomechanics involved. Physiotherapy Theory and Practice, 31 (7). pp. 518-526. ISSN 0959-3985

It is advisable to refer to the publisher's version if you intend to cite from the work. http://dx.doi.org/10.3109/09593985.2015.1037874

For more information about UCLan's research in this area go to http://www.uclan.ac.uk/researchgroups/ and search for <name of research Group>.

For information about Research generally at UCLan please go to http://www.uclan.ac.uk/research/

All outputs in CLoK are protected by Intellectual Property Rights law, including Copyright law. Copyright, IPR and Moral Rights for the works on this site are retained by the individual authors and/or other copyright owners. Terms and conditions for use of this material are defined in the policies page. 


\title{
Laserlight visual cueing device for freezing of gait in Parkinson's disease: a case study of the biomechanics involved
}

\author{
C. J. Egerton, MBChB, MSC (D', P. MCCandless, MSC, MCSP, SRP, FHEA ${ }^{2}$, B. Evans, BSc, MCSP, SRP1 ${ }^{2}$, J. Janssen, BHealth MSC, PhD ${ }^{2}$, \\ and J. D. Richards, BEng, MSC, PhD ${ }^{2}$ \\ 'Faculty of Health and Medicine, Lancaster University, Lancaster, UK and ${ }^{2}$ Allied Health Research Unit, University of Central Lancashire, Preston, UK
}

\begin{abstract}
Background: Freezing of gait (FOG) is a serious gait disorder affecting up to two-thirds of people with Parkinson's disease (PD). Cueing has been explored as a method of generating motor execution using visual transverse lines on the floor. However, the impact of a laser light visual cue remains unclear. Objective: To determine the biomechanical effect of a laser cane on FOG in a participant with PD compared to a healthy age- and gender-matched control. Methods: The participant with PD and healthy control were given a task of initiating gait from standing. Electromyography (EMG) data were collected from the tibialis anterior (TA) and the medial gastrocnemius (GS) muscles using an 8-channel system. A 10-camera system (Qualisys) recorded movement in 6 degrees of freedom and a calibrated anatomical system technique was used to construct a full body model. Center of mass (COM) and center of pressure (COP) were the main outcome measures. Results: The uncued condition showed that separation of COM and COP took longer and was of smaller magnitude than the cued condition. EMG activity revealed prolonged activation of GS, with little to no TA activity. The cued condition showed earlier COM and COP separation. There was reduced fluctuation in GS, with abnormal, early bursts of TA activity. Step length improved in the cued condition compared to the uncued condition. Conclusion: Laserlight visual cueing improved step length beyond a non-cued condition for this patient indicating improved posture and muscle control.
\end{abstract}

\section{Keywords}

EMG, gait, kinematics, kinetics, Parkinson's disease

\section{History}

Received 25 March 2014

Revised 24 January 2015

Accepted 14 February 2015

Published online 8 September 2015

\section{Introduction}

In the early $1800 \mathrm{~s}$, Sir James Parkinson attributed a stooped posture, resting tremor, and festinating gait to the condition we now know as Parkinson's Disease (PD) (Yanagisawa, 2006). However, it was not until 1926 that the phenomenon "freezing of gait" (FOG) was fitst documented by Gerstmann and Schilder (1926). FOG has been defined as "an episodic, brief inability to step that delays gait initiation or interrupts ongoing gait" (Jacobs et $\mathrm{al}, 2009$ ). It has been linked with depression, social isolation and a reduced quality of life (Giladi and Hausdorff, 2006; Moore, Peretz, and Giladi, 2007).

FOG is believed to affect between 30 and $60 \%$ of patients with PD (Giladi et al, 1992; Lamberti et al, 1997). Findings indicate that more frequent and prolonged episodes occur in the advanced stages, which can lead to falls (Dietz, 1997; Jankovic, 2009). Cardinal features include freezing when turning or passing through a narrow opening, initiating gait, as well as situations where there is pressure on the individual to move, for example at a set of traffic lights (Okuma, 2006). There is also a reported accompanying sensation of feeling "glued to the ground" (Okuma, 2006).

Address correspondence to: Chloe Egerton, Faculty of Health and Medicine, Lancaster University, Lancaster, UK. E-mail: c.egerton1@lancaster.ac.uk
Muscle activity, center of mass (COM), and center of pressure (COP) are important in gait. COM has been defined as "the point where all acting gravity forces are applied over the body". while COP has been given a definition of "the point where vertical forces are applied in reaction to the floor" (Merello, Fantacone, and Balej, 2010). This means that COM is a point where all mass can act, and is affected by both the shape and density distribution of the object or person, whereas COP refers to the average pressure point beneath the foot (Richards, 2008). Normal gait initiation relies on the vertical and lateral displacement of COM, and an increase in the displacement or separation from COP (Orendurff et al, 2004). PD patients have difficulty in aligning COM in relation to their COP, which requires certain postural adjustments (Merello, Fantacone, and Balej, 2010). Hass et al (2005) explored balance in individuals with PD with the use of COP-COM and found that individuals with impaired postural control produced shorter COP-COM distances than individuals without clinically detectable balance impairment. Furthermore, PD patients were found to have greater antero-posterior sway regardless of their medication state in comparison to healthy controls, making them more susceptible to falls (Menant et al, 2011). EMG data on gait in PD have shown that phasing of muscle activation patterns during the stride cycle is maintained, particularly in the distal lower limb muscles, which demonstrate reduced amplitude of gastrocnemius (GS) activity during the stance phase (Cioni et al, 1997; Dietz, Leenders, and Colombo, 1997). In addition, studies of gait initiation by healthy subjects 
show lateral COP displacement during preloading of the stepping foot by the hip abductors, and the backward COP displacement occurs due to activation of the tibialis anterior (TA), and deactivation of bilateral GS and soleus muscles (Halliday et al, 1998; Vaugoyeau, Viallet, Mesure, and Massion, 2003).

Various visual cues have been tested on PD patients experiencing FOG. Azulay, Mesure, and Blin (2006) suggested that using horizontal stripes across the floor could improve response. This concept has been further developed in the form of a laserlight cueing device, which consists of an inbuilt laser beam within a walking device designed to be activated during FOG. Although Lowry, Carrel, Mcilrath, and Smiley-Oye (2010) found minimal improvement using visual lines, Donovan et al (2011) tested patients using a laserlight cane for 1 month, which resulted in a significant mean reduction in the FOG Questionnaire. The biomechanical effects of visual cueing in PD patients experiencing FOG is less established. Some studies have characterized gait kinetics and kinematics in patients with PD (Morris, Iansek, Matyas, and Summers, 1998) demonstrating a degree of normalization with the addition of transverse lines (Morris et al, 1999). Similarly, in a study of gait initiation in $\mathrm{PD}$, gait velocity, push-off force and magnitude of first and second step length were significantly greater when transverse line visual cues were implemented (Jiang and Norman, 2006).

The focus of this case study was to investigate the effects of a laserlight visual cue on FOG. There are few studies investigating the use of a laserlight cue on FOG in a controlled environment, examining the impact of a visual cue on EMG measurements, $\mathrm{COM}, \mathrm{COP}$ and gait velocities, which may give important information about the motor adjustments taking place during cueing and the application of laserlight cues in a community setting.

\section{Method}

\section{Participants}

This study was approved by the University of Central Lancashire Ethics Committee and NHS ethics committee. A single participant with PD was a 60-year-old male who was diagnosed with PD by a neurologist 10 years ago. Before the trial commenced, any additional musculoskeletal, sensory or motor impairments were inquired about that would affect gait. A validated severity scale, the Unified Parkinson's Disease Rating Scale (UPDRS) was completed by the participant using part 2 (self-evaluation of the activities of daily life) and part 3 of the UPDRS (evaluation of motor function) (Fahn and Elton, 1987). Data were collected when the participant was in the OFF state, in which he scored 41 on part 3 of the UPDRS (in the ON state the participant scored 23). The OFF state was determined by withholding the dose of L-dopa prior to testing, which approximated to 12 hours without medication overnight. This was done to reduce the chances of capturing L-dopa induced dyskinesia, which is substantially lessened when plasma levels of L-dopa are low, such as in the morning (Thanvi, Lo, and Robinson, 2007). In addition, a healthy control, who was recruited from the University of Central Lancashire, was matched for age and gender, with no documented neurological or musculoskeletal problems. (a)

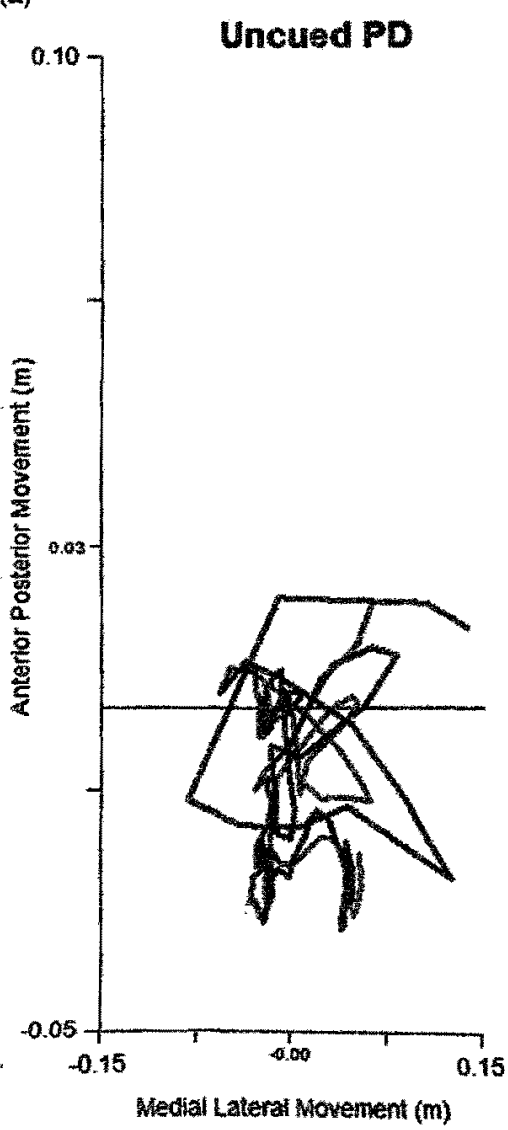

\section{Paths of COM and COP during gait initiation}

(b)

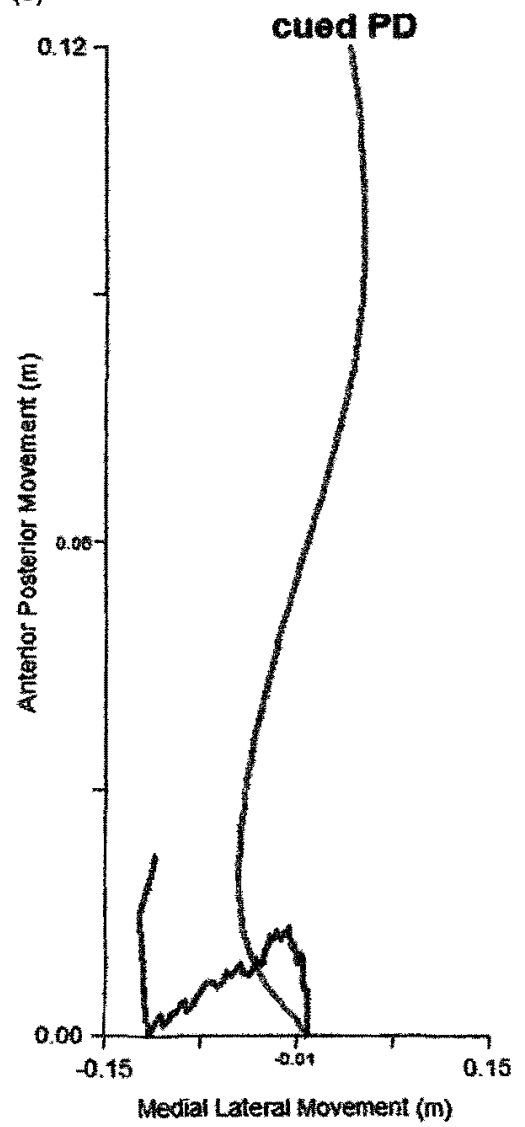

(c)

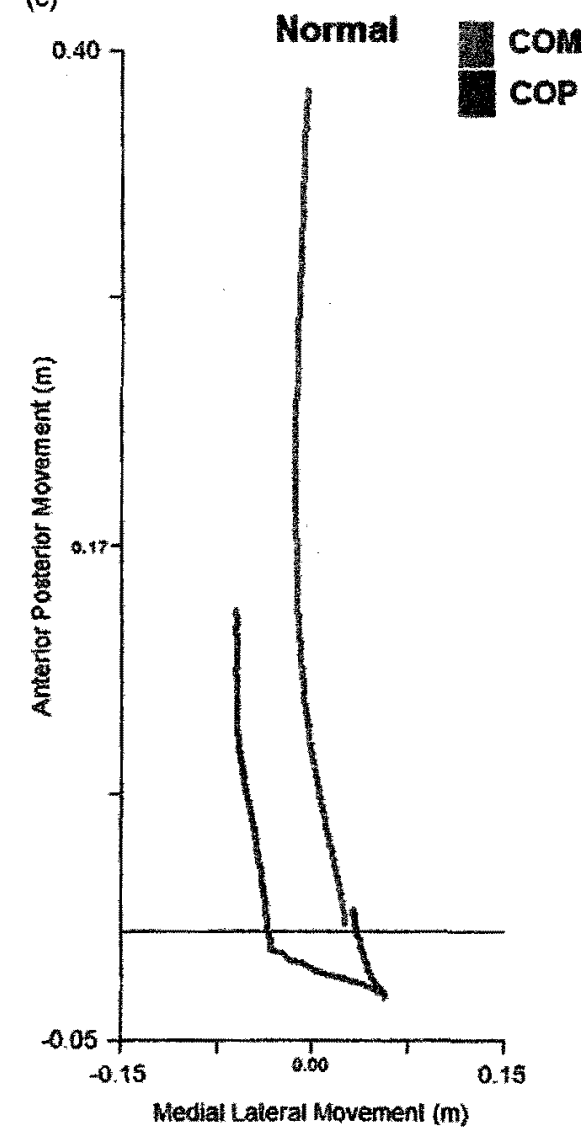

Figure 1. COM and COP pathway in a PD patient and heal thy control. Recordings were taken during a single stride with the right foot from a stationary position. Recordings for the PD patient were captared during a FOG episode. (a) PD patient without a lasercane. (b) PD patient with a lasercane. (c) Healthy control without a lasercane. The horizontal line represents the start position of the participant in relation to the lab. 
Both participants gave written informed consent according to the declaration of Helsinki before entering the study.

\section{Conditions}

The participants were given the task of initiating gait after assuming a stable stationary position. The participant with PD was shown how the lasercane ${ }^{\mathrm{TM}}$ projects a visible line when a small amount of downward force is applied, however no other information was given regarding the potential benefits. The participant with PD was instructed to walk forwards initially without a visual cue and then with a visual cue using a lasercane ${ }^{\mathrm{TM}}$, whereas the healthy control was only tested without a visual cue. Both participants started from a seated position and were asked to stand with each foot on a separate force plate. After 5 seconds they were asked to start walking in their own time, but care was taken to ensure that there was sufficient delay so that the instruction did not itself act as a cue. The preference of striding foot was not controlled as this would have created undue difficulties for the patient and may have interfered with the initiation.

\section{Procedures}

Data were collected from three trials under each condition. Force data were collected through three AMTI plates, two platforms side by side and a third platform solely to detect heel-swing contact (Advanced Mechanical Technology, Inc, Water-town, MA).
For movement data, a 10-camera Oqus System (Qualisys Medical AM, Gothenburg, Sweden) was used. Calibration of the Oqus system was performed before each data collection session. Electromyography (EMG) data were collected from the tibialis anterior (TA) and medial GS muscles on the left and right side using 4 DE-2.1 single differential surface EMG sensors with a fixed $10 \mathrm{~mm}$ electrode distance placed over the belly of the muscle. These were recorded through a Bagnoli system with preamplified gain of 1000 (Delsys, Inc, Boston, MA). Alcohol wipes were used to clean and lightly abrade the skin surface prior to attachment of the electrodes. The EMG signals were examined visually for any obvious data quality issues or cross talk prior to commencing data collection. Force and $\mathrm{EMG}$ data were attained using a 16-bit analog-to-digital converter at $2000 \mathrm{~Hz}$.

\section{Modeling}

CAST (calibrated anatomical systems technique) was used to produce a 12 segment model of the full body in 6 degrees of freedom. Prominent anatomical landmarks were used in order to provide a point of reference. Landmarks used included the acromion, lateral condyle of the humerus, ulnar styloid process, anterior and posterior iliac spines on the pelvis, greater trochanter, medial and later epicondyles of the femur, and the medial and later malleoli. The segments used consisted of 4 marker clusters attached to a plastic shell. A calibration test collected data for 1 second which was carried out with the patient in a standing position. This enabled the identification of the precise location of (a)

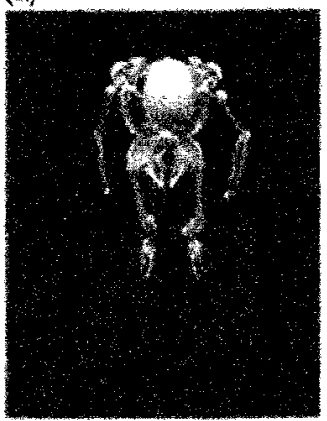

(d)

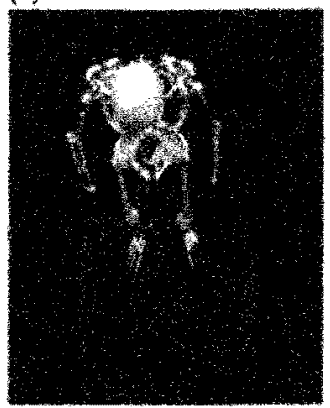

(b)

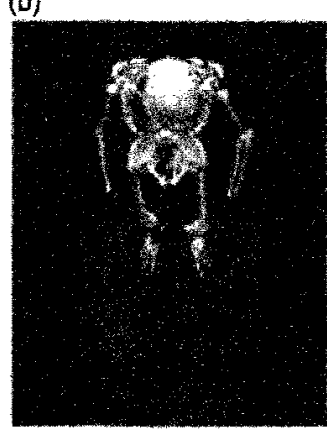

(e)

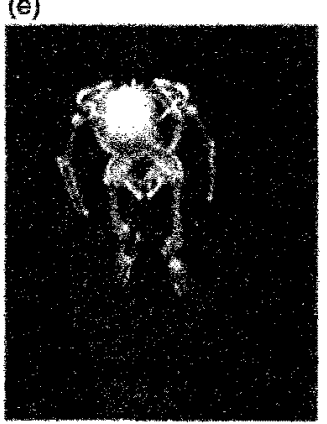

(c)

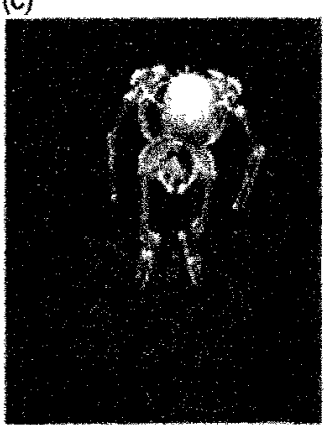

(1)

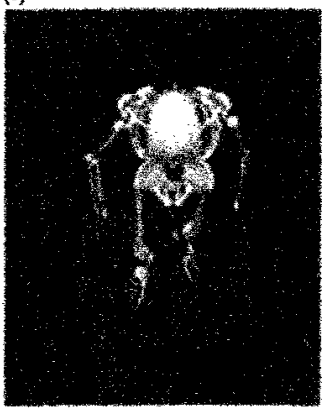

(g)

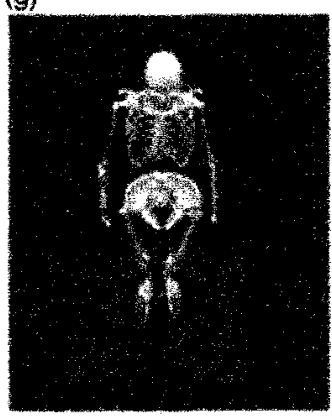

(h)
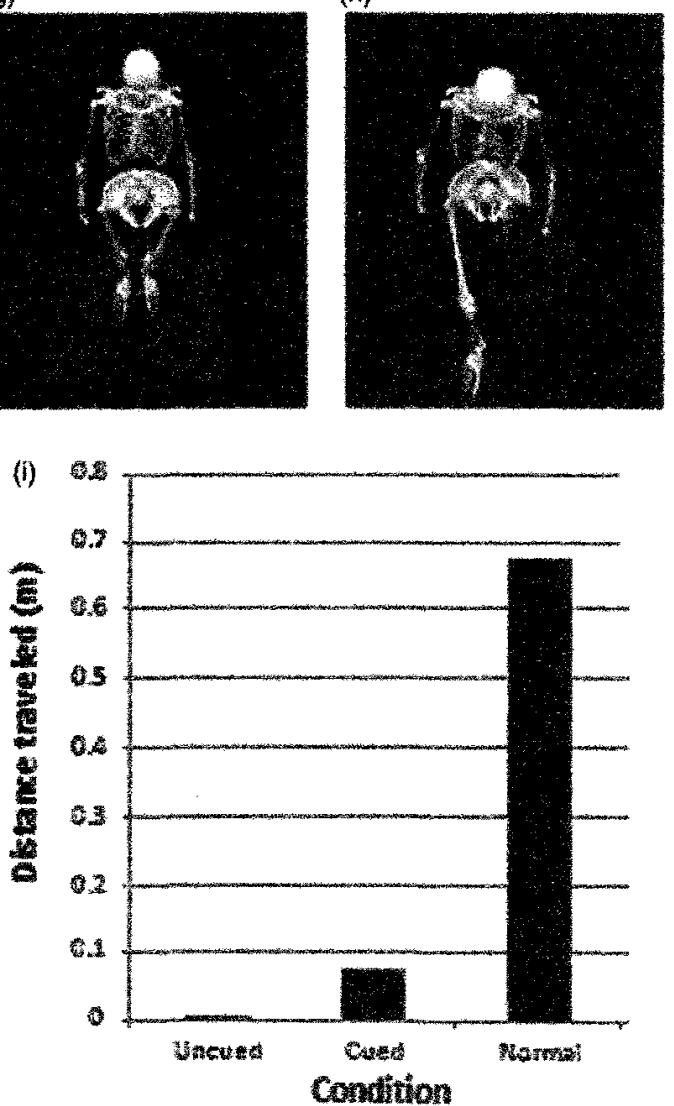

Figure 2. Visual 3D construction of a PD patient and healthy control initiating gait. Yellow circle represents COM and blue arrow represents COP. (ac) Capture a FOG episode without the use of a lasercane. $(\mathrm{d}-\mathrm{f})$ Capture a FOG episode with the use of a lasercane. (g) and (h) Capture a healthy control initiating gait. (a) PD patient prior to initiating gait. (b) PD patient upon contact of first step. (c) PD patient upon contact of second step. (d) PD patient prior to initiating gait. (e) PD patient upon contact of first step. (f) PD patient upon contact of second step. (g) Healthy control prior to initiating gait. (h) Healthy control upon contact of first step. (i) A graph to show the distance traveled after first step of gait initiation. 
each segment, and provided local coordinate systems for all segments on the model. The $x$-axis equaled the medial-lateral, the $y$-axis equaled the anterior-posterior, and the $z$-axis equaled the proximal-distal planes. The median distances between the medial and lateral joint markers were used to calculate the centers of the elbow, wrist, knee and ankle joints. Pelvic depth and width were used to calculate the center of the hip joint, using Bell, Pedersen, and Brand (1990) regression coefficient. A Cardan method with an $X Y Z$ order of rotations was used to calculate joint kinematics.

\section{Data processing}

Visual 3D (C-motion, Inc, Germantown, MD) was used after the raw force and movement data had been exported for processing. A fourth-order, low-pass Butterworth filter with cut-off frequencies of $6 \mathrm{~Hz}$ and $25 \mathrm{~Hz}$ was used on motion and force data, respectively. EMG data were full-wave rectified and enveloped using a fourth-order, low-pass Butterworth filter with a cut-off frequency of $25 \mathrm{~Hz}$. Visual $3 \mathrm{D}$ was also used to calculate COP by combining the data from the two force plates, one under each foot during the initiation phase, and to calculate the position of the
COM from the full body model in the medial-lateral and anteriorposterior directions.

\section{Data extraction}

The variables measured included COM and COP pathways, providing information on anterior-posterior and medial-lateral movement measured in meters $(\mathrm{m})$. The next variable measured was step length, which was the distance traveled after taking one step (m). A visual 3D construction was also used to calculate COP but also to better visualize the gait initiation process. COM velocity was also measured $(\mathrm{m} / \mathrm{s})$ in both the anterior-posterior and medial-lateral planes, as a percentage of first step time. The final variable measured was EMG activity in the GS and TA measured in millivolts ( $\mathrm{mV}$ ) of the stance and step legs as percentage of first step time. Each variable was measured across 3 conditions, the PD uncued condition, PD cued condition, and an age- and gendermatched control condition. All data collected in the uncued trials resulted in a FOG episode, which was defined as the inability to take a step within the first 10 seconds after standing. Values were averaged across all three trials to determine distance traveled, and representative graphs were selected for the other variables.

\section{Medial-lateral COM velocity during gait initiation}

(a)

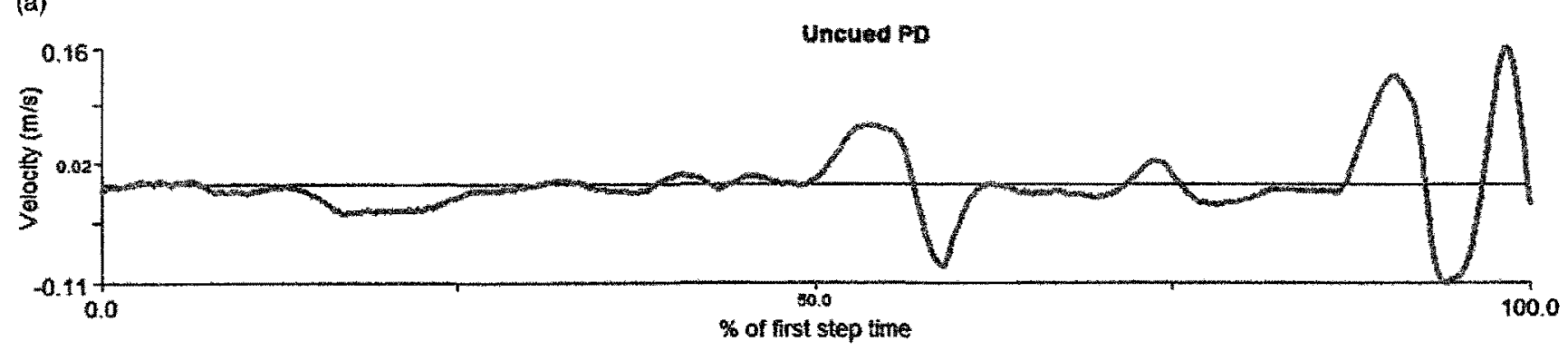

(b)

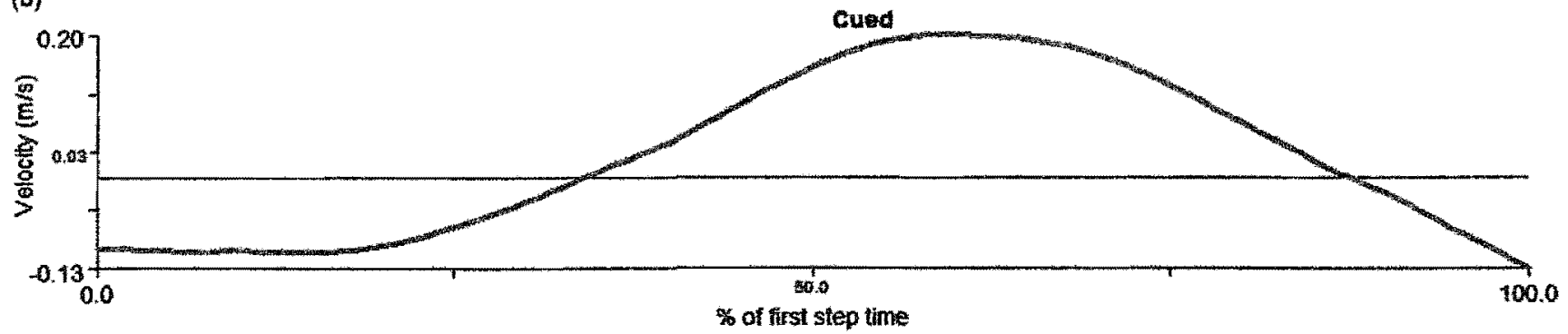

(c)

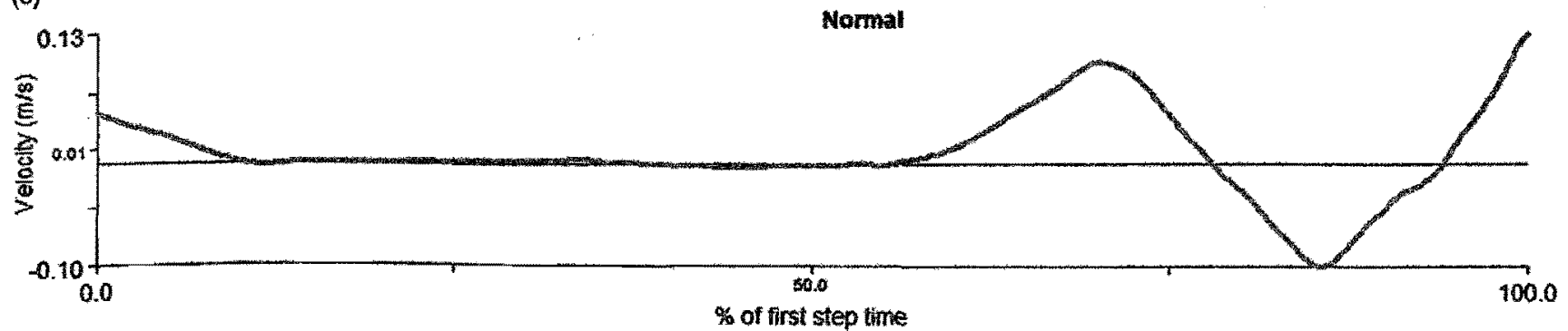

Figure 3. Velocity of $\mathrm{COM}$ (VCOM) during the initiation of gait. Recordings of PD patient were taken during a FOG episode. (a) Medial-1ateral vCOM in PD patient without lasercane. (b) Medial-lateral VCOM in PD patient with lasercane. (c) Medial-lateral vCOM in healthy control. (d) Anteroposterior $\mathrm{VCOM}$ in PD patient without lasercane. (e) Antero-posterior vCOM in PD patient with lasercane. (O) Antero-posterior vCOM in healthy control. 

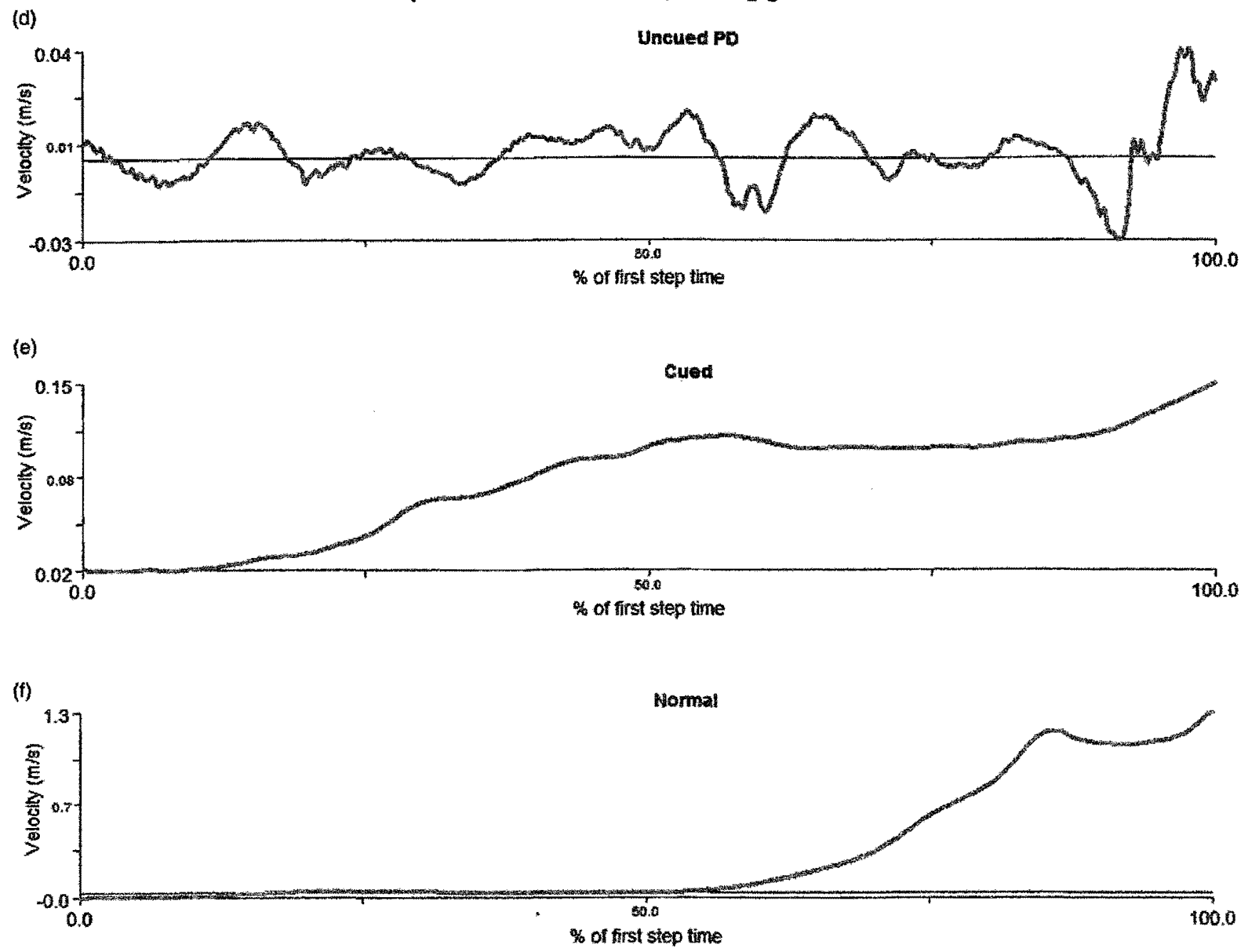

Figure 3. Continued.

\section{Results}

The focus of this case study was to investigate the effects of a lasercane visual cue on FOG, measuring COM, COP, COM velocity, EMG activity, and drawing comparisons with an ageand gender-matched control.

\section{COM and COP pathway}

The PD uncued condition shows a failure of the COM and COP positions, as well as posterior displacement of COM and COP. The COM and COP pathway also terminates prematurely in comparison to the control and cued condition, with minimal shift in the anterior plane (Figure 1a). In the PD cued condition, the COP shows a more defined, earlier separation from COM when compared to the uncued pathway, despite a lack of posterior displacement when compared with the control, along with a lateral displacement of COM in the direction of the COP (Figure $\mathrm{lb}$ ). In the healthy control, COM and COP showed a normal relationship whereby the $\mathrm{COM}$ and $\mathrm{COP}$ separated smoothly at the beginning of gait initiation (Figure lc).

\section{Visual 3D construction and walking distance}

The visual 3D construction images further illustrate the pathways of COM and COP. In the PD uncued condition the distance traveled was $0.004 \mathrm{~m}$ (Figures $2 \mathrm{a}-\mathrm{c}$ ), which increased to $0.075 \mathrm{~m}$ in PD cued condition (Figures $2 \mathrm{~d}-\mathbf{f}$ ). For the healthy control, distance achieved after the first stride was $0.674 \mathrm{~m}$ (Figures $2 \mathrm{~g}-\mathrm{h}$ ). The addition of a lasercane resulted in over an 18 fold improvement (Figure 2i). Although the first two steps for the PD subject for the cued and uncued conditions are shown, the data were only analyzed for the first step.

\section{Velocity of COM}

The medial-lateral velocity of COM (VCOM) in the uncued condition showed abnormal fluctuations throughout, followed by a premature and diminished increase in lateral velocity, and a reciprocal pattern of vCOM with minimal anterior acceleration in the antero-posterior direction (Figures $3 \mathrm{a}$ and $3 \mathrm{~d}$ ). The cued condition showed fewer overall fluctuations than the uncued condition, accompanied by an early acceleration in lateral $\mathrm{vCOM}$, but with a greater maximum velocity than in the uncued condition (Figure 3b). The cued condition in comparison to the normal also demonstrates a prolonged medial displacement of $\mathrm{VCOM}$ as it returns to the midline upon contact of first step. When compared to the healthy control, the cued anterior VCOM shows earliet acceleration over a more prolonged portion of gait initiation (Figure 3e). The $\mathrm{VCOM}$ in the healthy control shows a normal medial-lateral and antero-posterior pattern (Figures $3 \mathrm{c}$ and $3 \mathrm{f}$ ). 
(a) EMG during Gait Initiation in a PD Patient with Freezing Gait
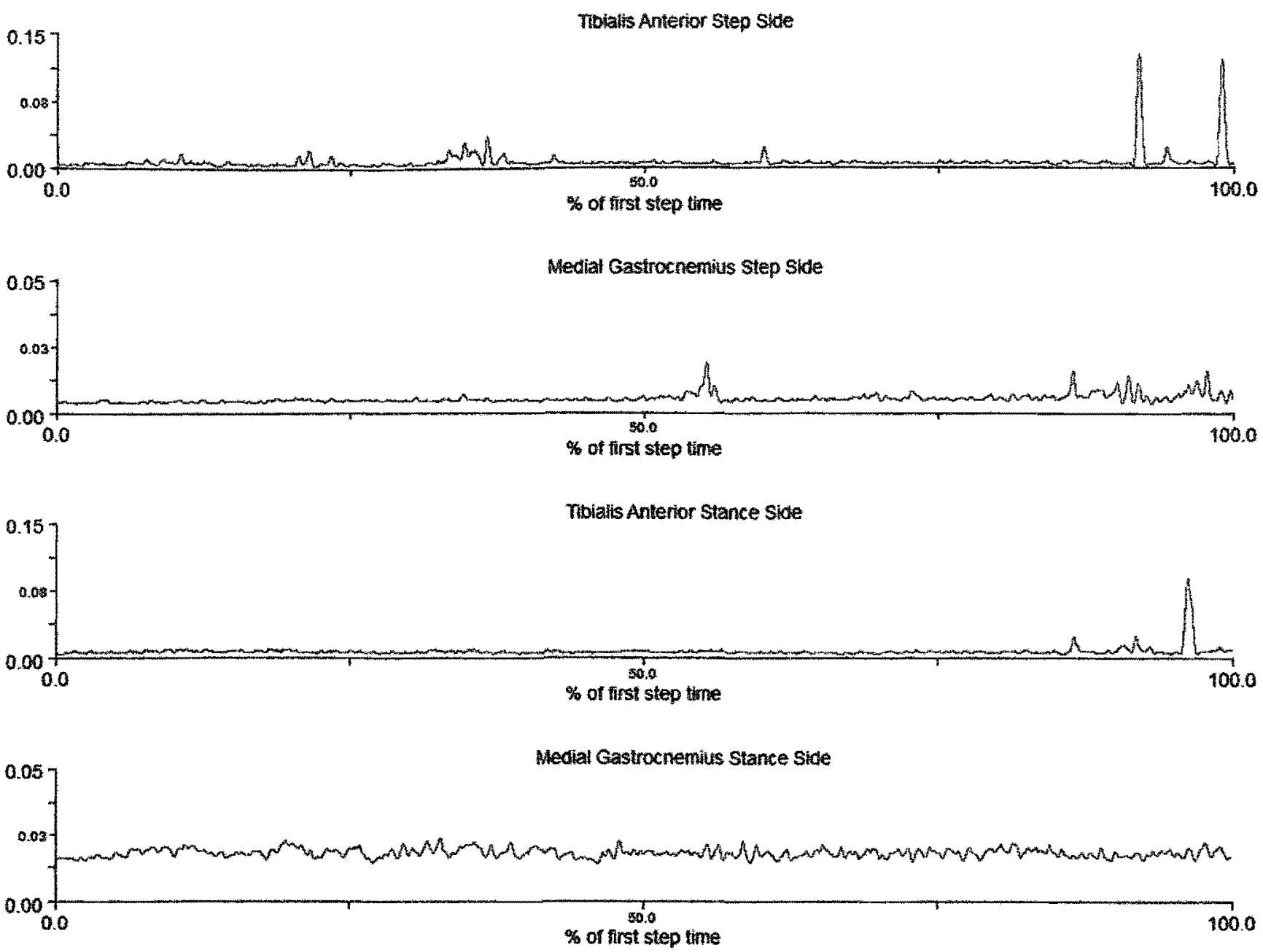

Figure 4. RMS EMG activity taken from the gastrocnemius (GS) and tibialis anterior (TA) of stance and swing leg during gait initiation. $x$-axis represents percentage of gait initiation. $y$-axis presents voltage $(\mathrm{mV})$. (a) Data taken from PD patient during a FOG episode without a lasercane. (b) Data taken from PD patient during a FOG episode with a lasercane. (c) Data taken from healthy control. EMG activity is presented for Step Side TA, Step Side GS, Stance Side TA, Stance Side GS.

\section{EMG activity}

No obvious cross talk was seen in either participant between TA and GS prior to data collection. The uncued condition showed little muscle activity in the GS, which is more pronounced in the step side. There is also abnormal activity in the GS of the step limb which occurs just after the midway point. Muscle activity in the TA is vastly reduced with little to no indication of a toe-off point. Timing of the limited TA activity is also toward the end of the cycle (Figure 4a). The cued condition shows reduced fluctuations in GS activity, along with early GS activation in the stance limb and reduced force of contraction. TA activity in the stance limb occurs later than in the healthy control, but earlier in the step limb, showing early unilateral activation and deactivation (Figure $4 b$ ). EMG activity in the healthy control shows a phasic pattern of TA and GS activity during gait initiation (Figure 4c).

\section{Discussion}

This case study set out to explore the biomechanical effects of a laserlight visual cue on FOG in a patient with $P D . C O M$ and $C O P$ pathway showed an impaired separation of COM and COP positions in the PD patient, whereby normal gait is initiated when the "COM decouples or separates from the COP causing the body to fall forward about the ankle joint" (Richards, 2008). The small fluctuations in COP pathway in the uncued condition indicates difficulties surrounding balance that were encountered along with failed attempts at initiating gait. There is also a reduced COMCOP distance in the uncued condition compared to the cued condition and healthy control (Figure 1). Hass et al (2005) in a study of 43 participants found that shorter COM-COP distances correlated to $\mathrm{PD}$ patients with greater postural and motor impairment. The data in Figure 1 show that the cued condition is able to increase COM-COP distance compared to the uncued, indicating potential application of a lasercane for severely affected patients. However, there remains a lack of posterior COP displacement in the cued condition as demonstrated by the control, which may be due to a fall forward to initiate gait. Larger studies would be useful to determine how a lasercane affects these pathways and whether this pattern would differ between PD patients using a lasercane.

With regard to vCOM, the uncued condition showed small fluctuations, reduced velocity, and an abnormal pathway which correlated to the reduction in step length shown in Figure 2(i). Similarly, Gantchev, Viallet, Aurenty, and Massion (1996) found PD patients with FOG to have a decreased vCOM, and a reduced 
(b)

\section{EMG during Gait Initiation in PD Patient with Visual Cue}
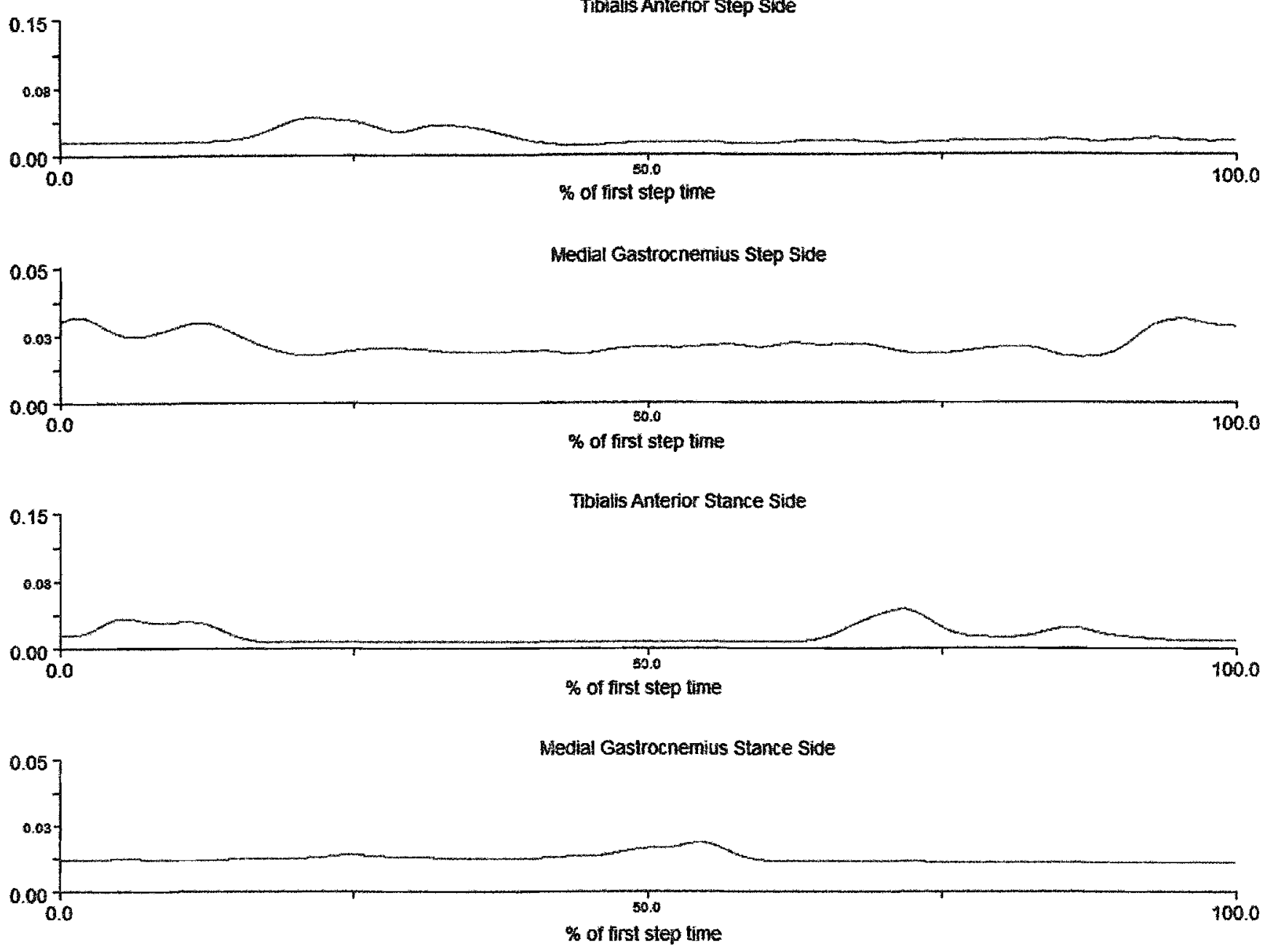

Figure 4. Continued.

maximum velocity of the COM, contributing to a smaller step length. The cued condition, although abnormal in comparison to the control, showed a greater lateral displacement in vCOM which occurred more quickly than in the uncued condition, along with fewer fluctuations. Elble, Cousins, Leffler, and Hughes (1996) found that PD patients who had a greater lateral shift of vCOM toward the stance foot enabled a more forward projection of the body. The results from this study agree with those found by Elble, Cousins, Leffler, and Hughes (1996) as a greater lateral vCOM coincided with increased step length (Figure 2i), and increased anterior displacement (Figure 3e).

Muscle activity investigated in the PD patients demonstrates a lack of smooth coordination between the GS and TA. The uncued condition showed abnormal activation of the GS, and there was also a bilateral decrease in TA activity, with delayed action and reduced maximal contraction. In the study by Elble, Cousins, Leffler, and Hughes (1996), EMG findings also showed prolonged GS activity. Disturbed organization of GS and TA activity was reported by Nieuwboer (2006). EMG showed a reduction in sustained GS activity, indicating improved control of the lower limbs in initiating gait. Investigations have attributed prolonged GS activity to tonic plantar flexion around the ankle joint, which compensates for the tendency of patients to lean forward, which may explain ongoing GS activity in these results, particularly in the uncued condition (Elble, Cousins, Leffler, and Hughes, 1996).
Reduced frequency of GS contractions in the cued condition indicates the patient is making central postural adjustments which reduce GS activity, heightened by the use of a laserlight visual cue. Further comparisons with other studies of a visual laserlight cue are difficult to draw, as Donovan et al (2011) focused on the response from questionnaires filled out by participants. The reduction in falls as confirmed by the participants from the Donovan et al (2011) study is supported by biomechanical findings from this single case study, whereby use of a lasercane facilitates postural alterations which are conducive to more effective and coordinated gait initiation.

The neurophysiological mechanisms responsible for the improvements observed following a laserlight visual cueing device have several possibilities. It has been postulated that visual cueing devices enhance optical flow, suggesting that PD patients are more dependent on visual feedback (Azulay, Mesure, and Blin, 2006). Moreover, the ability of visual cueing to focus attention on the stepping process has been pointed out, with greater success observed in studies that did not require unnatural activation of the cue, which could divert attention away from the primary task (Bunting-Perry et al, 2013). In addition, Morris et al (2001) measured cerebral blood flow and hypothesised that patients could compensate for basal-ganglia dysfunction by using non-affected areas of the brain for motion. Indeed, the pre-motor cortex and supplementary motor area have shown increased 

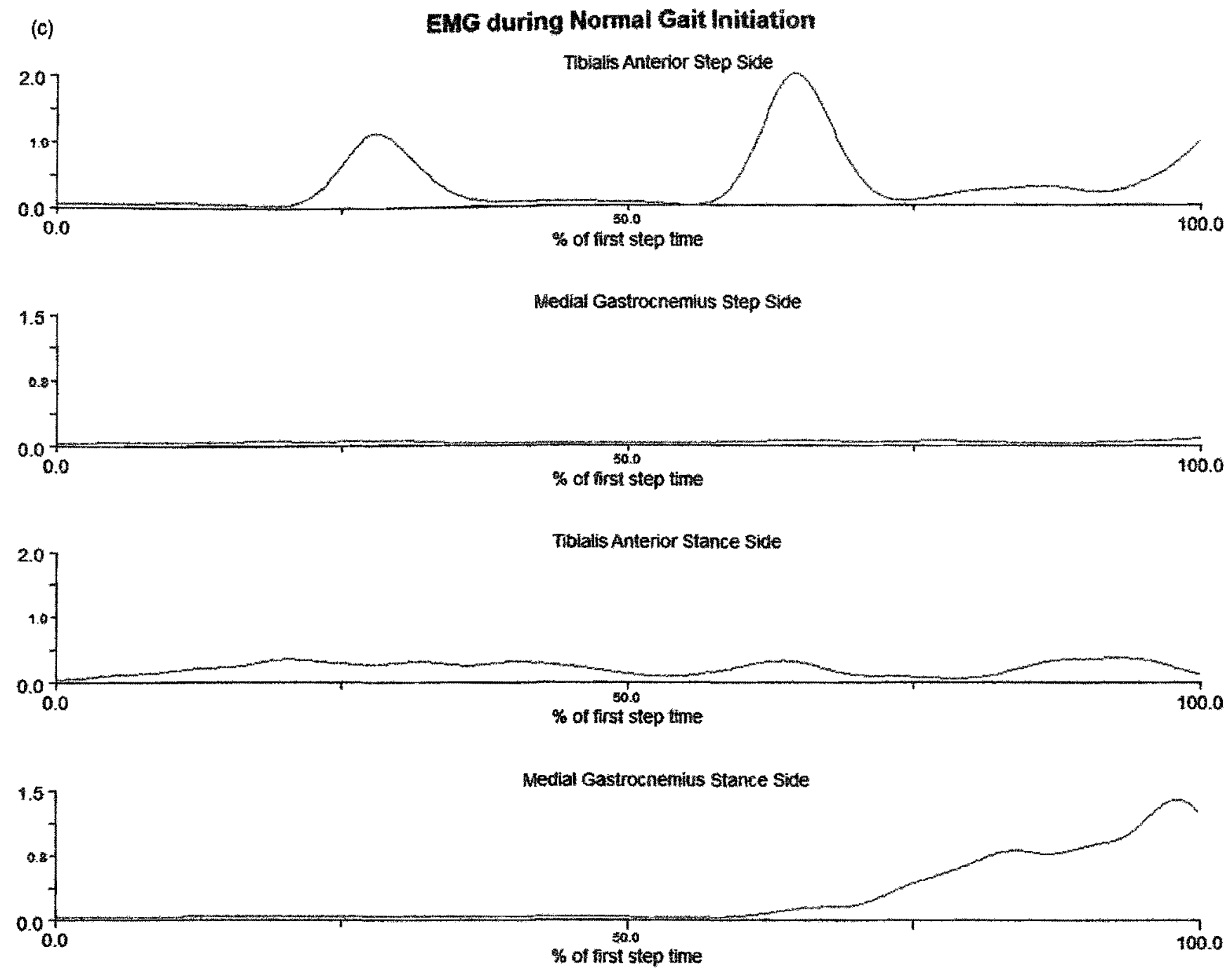

Figure 4. Continued.

activity in $\mathrm{PD}$ patients attempting motor execution following exposure to external cues (Jueptner and Weiller, 1998). Findings from the present research similarly indicated that nearer-normal patterns of movement are not lost with PD patients, but perhaps there exists an activation problem. With further study it may become apparent as to the extent a lasercane can aid in reactivating movement.

In comparison to the uncued condition, the laserlight cue altered the pattern of muscle activity, increased lateral and anterior $\mathrm{YCOM}$ displacement, resulting in a greater step length. If other patients can adapt their postural habits using a lasercane, as implied by the EMG, COM and COP results, this could lead to a reduction in the frequency and severity of falls as caused by a lack of postural adjustment which often makes falling more severe. This single case study has demonstrated that the patient was able to synchronise movements with the visual cue, rather than becoming distracted by it.

To conclude, a laserlight visual cue could be used as an external aid to temporarily overcome internal neuronal dysfunctions associated with FOG. What has been shown is that difficulty initiating gait during a FOG episode cannot be completely eliminated with a lasercane. However, it can produce results which support better gait execution when compared to an uncued FOG episode. This study was limited to one case in the OFF state just prior to taking their medication, whereby a larger patient study would be useful, both within and outside a clinical setting. More studies which capture FOG are needed to assess the use of a lasercane intervention, along with research to determine patient satisfaction and suitability using this device. The present results may not apply to every individual patient with FOG, as symptoms, severity and management will vary, but it could provide a portable, tangible tool readily transferable to everyday situations.

\section{ORCID}

\section{J. Egerton D htp://orcid.org/0000-0003-1919-5955}

\section{Declaration of interest}

The authors report no declaration of interest.

\section{References}

Azulay JP, Mesure S, Blin 02006 Influence of visual cues on gait in Parkinson's disease: Contribution to attention or sensory dependence? Journal of the Neurological Sciences 248: 192-195.

Bell AL, Pedersen DR, Brand RA $1990 \mathrm{~A}$ comparison of the accuracy of several hip center location prediction methods. Journal of Biomechanics 23: 617-621.

Bunting-Perry L, Spindler M, Robinson KM, Noorigian J, Cianci HJ, Duda JE 2013 Laser light visual cueing for freezing of gait in 
Parkinson disease: A pilot study with male participants. Journal of Rehabilitation Research and Development 50: 223-230.

Cioni M, Richards CL, Malouin F, Bedard PJ, Lemieux R 1997 Characteristics of the electromyographic patterns of lower limb muscles during git in patients with Parkinson's disease when OFF and ON L-Dopa treatment. Italian Journal of Neurological Sciences 18 : and $195-208$.

Dietz V 1997 Neurophysiology of gait disorders: Present and future applications. Electroencephalography and Clinical Neurophysiology 103: 333-355.

Dietz V, Leenders KL, Colombo G 1997 Leg muscle activation during gait in Parkinson's disease: Influence of body unloading. Electroencephalography and Clinical Neurophysiology 105: 400-405.

Donovan S, Lim C, Diaz N, Browner N, Rose P, Sudarsky LR, Tarsy D, Fahn S, Simon DK 2011 Laserlight cues for gait freezing in Parkinson's disease: an open-label study. Parkinsonism and Related Disorders 17: 240-245.

Elble RJ, Cousins R, Leffler K, Hughes L 1996 Gait initiation by patients with lowerhalf parkinsonism. Brain 119: 1705-1716.

Fahn S, Elton RL UPDRS Development Committee. 1987 The unified Parkinson's disease rating scale. In: Fahn S, Marsden CD, Calne DB, Parkinson's disease rating scale. In: Fahn $S$, Marsden $C D$, Calne DB,
Goldstein $M$ (eds) Recent developments in Parkinson's disease. 2nd ed, pp 153-163. Macmillan Healthcare Information, Florham Park.

Gantchev N, Viallet F, Aurenty R, Massion I 1996 Impairement of posturo-kinetic co-ordination of forward oriented stepping movements in parkinsonian patients. Electroencephalography and Clinical Neurophysiology 101: 110-120.

Gerstmann J, Schilder P 1926 Uber eine besondere gangstörung bei stimhimerkrankung. Wien Med Wschr 76: 97-102.

Giladi N, Hausdorff JM 2006 The role of mental function in the pathogenesis of freezing of gait in Parkinson's disease. Journal of the Nenrological Sciences 248: 173-176.

Giladi N, Mcmahon D, Przedborski S, Flaster E, Guillory S, Kostic V, Fahn S 1992 Motor blocks in Parkinson's disease. Neurology 42: 333-339.

Halliday SE, Winter DA. Frank JS, Patla AE, Prince F 1998 The initiation of gait in young, elderly, and Parkinson's disease subjects. Gait and Posture 8: 8-14.

Hass CJ, Waddell DE, Fleming RP, Juncos IL, Gregor RJ 2005 Gait initiation and dynamic balance control in Parkinson's disease. Archives of Physical Medicine and Rehabilitation 86: 2172-2176.

Jacobs JV, Nutt JG, Carlson-Kuhta P, Stephens M, Horak FB 2009 Knee trembling during freezing of gait represents multiple anticipatory postural adjustments. Experimental Neurology $215 ; 334-341$.

Jankovic J 2009 Atomoxetine for freezing of gait in Parkinson disease. Joumal of the Neurological Sciences 284: 177-178.

Jiang Y, Norman KE 2006 Effects of visual and auditory cues on gai initiation in people with Parkinson's disease. Clinical Rehabilitation 20: $36-45$.
Jueptner M, Weiller C 1998 A review of differences between basal ganglia and cerebellar control of movements as revealed by functional imaging studies. Brain 121: 1437-1449.

Lamberti $P$, Armenise S, Castaldo V, De Mari M, Iliceto $G$, Tronci $P$ Serlenga L 1997 Freezing gait in Parkinson's disease. European Neurology 38: 297-301.

Lowry KA, Carrel AJ, Mcilrath JM, Smiley-Oyen AL 2010 Use of harmonic ratios to examine the effect of cueing strategies on gait stability in persons with Parkinson's disease. Archives of Physical Medicine and Rehabilitation 91: 632-638.

Menant JC, Latt MD, Menz HB, Fung VS, Lord SR 2011 Postural sway approaches center of mass stability limits in Parkinson's disease. Movement Disorders 26: 637-643.

Merello M, Fantacone N, Balej J 2010 Kinematic study of whole body center of mass position during gait in Parkinson's disease patients with and without festination. Movement Disorders 25: 747-754.

Moore O, Peretz C, Giladi N 2007 Freezing of gait affects quality of life of peoples with Parkinson's disease beyond its relationships with mobility and gait. Movement Disorders 22: 2192-2195.

Morris ME. Huxham F, McGinley J, Dodd K, Iansek R 2001 The biomechanics and motor control of gait in Parkinson disease. Clinical Biomechanics 16: 459-470.

Morris ME, Jansek R, Matyas T, Sumners J 1998 Abnormalities in the stride length-cadence relation in parkinsonian gait Movement Disorders 13: 61-69.

Morris ME, McGinley J, Huxham F, Collier J, Iansek R 1999 Constraints on the kinetic, kinematic and spatiotemporal parameters of gait in Parkinson's disease. Human Movement Science 18: 461-483.

Nieuwboer A 2006 Freezing of gait: Problem analysis and rehabilitation strategies. Parkinsonism and Related Disorders 12: \$53-S54.

Okuma Y 2006 Freezing of gait in Parkinson's disease. Journal of Neurology 253: 27-32

Orendurff MS, Segal AD, Klute GK, Berge JS, Rohr ES, Kadel NJ 2004 The effect of walking speed on center of mass displacement. Journal of Rehabilitation Research and Development 41:829-834.

Richards I 2008 Biomechanics in clinic and research. Edinburgh Churchill Livingstone.

Thanvi B, Lo N, Robinson T 2007 Levodopa-induced dyskinesia in Parkinson's disease: Clinical features, pathogenesis, prevention and treatment. Postgraduate Medical Journal 83: 384-388.

Vaugoyeau $M$, Viallet $F$, Mesure S, Massion J 2003 Coordination of axial rotation and step execution: Deficits in Parkinson's disease. Gait and Posture 18: 150-157.

Yanagisawa N 2006 Natural history of Parkinson's disease: From dopamine to multiple system involvement. Parkinsonism and Related Disorders 12: $40-46$ 